\title{
COMMENT
}

\section{Prolonged early antimicrobials in ELBWs: too much for too little}

\author{
Pediatric Research (2019) 85:929-930; https://doi.org/10.1038/s41390-019-0360-5
}

In 2009, Cotten and colleagues ${ }^{1}$ reported on 4039 extremely low birth weight (ELBW) infants cared for in the Eunice Kennedy Shriver National Institute of Child Health and Development (NICHD) Neonatal Research Network (NRN) from 1998 to 2001, and found an association between prolonged duration ( $\geq 5$ days) of initial empiric antibiotic treatment [in whom blood and cerebrospinal fluid (CSF) culture results were sterile] and increased rates of death and necrotizing enterocolitis (NEC) ${ }^{1}$. They showed "each empirical treatment day was associated with increased odds of death, necrotizing enterocolitis, and the composite measure of necrotizing enterocolitis or death." As with any retrospective cohort analysis, there were caveats and limitations. Still, this publication was among the first reports that demonstrated that prolonged early antimicrobial treatment may violate our duty to "first do no harm." Another report followed showing a greater risk for lateonset sepsis (LOS) and the combination of LOS, NEC, or death among very low birth weight infants who received prolonged courses of empiric antibiotics².

In this issue of Pediatric Research, Greenberg and colleagues ${ }^{3}$ examined a cohort of 5730 ELBW, 22-28-week gestation infants cared for in the Eunice Kennedy Shriver NICHD NRN from 2008 to 2014 with the intent to: (1) describe changes over time in prolonged early antibiotic therapy among infants without cultureconfirmed (blood or CSF) infection or an intra-abdominal inflammatory process [spontaneous intestinal perforation (SIP)/ $\mathrm{NEC}$, and (2) describe associations of prolonged early antibiotic therapy with adverse outcomes on a more contemporary cohort. Overall, they found that the rate of prolonged early antibiotics declined from $49 \%$ in 2008 to $35 \%$ in 2014 without a rise in death, LOS, or NEC. The decrease in exposure without an increase in risk represents a significant achievement and simultaneously demonstrates the critical need for further efforts to reduce prolonged antibiotic exposure, which was 10-100-fold greater than the rate culture-proven early-onset sepsis (EOS). Of course, bacteremia, meningitis, and SIP/NEC are not the only valid indications for prolonged antibiotics. Treatment of bacterial pneumonia with antibiotics is absolutely appropriate. The authors acknowledged the inability to identify babies who received prolonged early antibiotics for pneumonia and had negative blood/CSF cultures. At present, no study can effectively address this limitation, even prospectively in future trials, without consensus criteria for the definition of pneumonia in this population. Of note, a requirement for continuous mechanical ventilation (for the first 7 days of life; CMV) was present in $55 \%$ of those infants who received prolonged early antibiotics, but was also present in $33 \%$ of those who did not. Thus, although the need for mechanical ventilation can be associated with pneumonia, and is a reasonable surrogate for the severity of illness, the reasons why a large proportion of infants who exhibited similar mechanical ventilation requirements but did not receive prolonged early treatment remain unclear.
Babies who received prolonged early antibiotics were more likely to have a 5 -min Apgar score $<5$ ( 24 vs. $17 \%$ ), were smaller (730 vs. $790 \mathrm{~g}$ ), less mature ( 25 vs. 26 weeks), less likely to have been born by Cesarean section (64 vs. 73\%), but more likely to have been exposed to both antenatal antibiotics (76 vs. $71 \%$ ) and rupture of membranes $>24 \mathrm{~h}$ ( $28 \mathrm{vs}$. $18 \%$ ) compared to those infants who did not receive prolonged early antibiotics. Collectively, these data suggest prolonged early treatment is heavily weighted to a symptomatic population of the most premature ELBWs with increased risk factors for, but not confirmed, EOS. It is unclear what criteria were used to support the decision to treat these infants as infected for 5 or more days at each respective center, but it is likely that those criteria are variable. This conclusion is supported by the substantial variation (30-69\%) shown in the proportion of infants who received prolonged early antibiotics unrelated to the site incidence of EOS among NICHD NRN centers (27-85\% in the 2009 Cotten et al. report). Strikingly, these data show that the place of birth could be associated with up to a 7-fold higher risk of prolonged early antibiotic exposure in the absence of EOS even after adjustment for demographic and clinical factors.

In contrast to the prior NRN study, no statistically significant associations for death (adjusted odds ratio (aOR) 1.17; 95\% confidence interval (Cl) 0.99-1.40, $p=0.07$ ), NEC (aOR 0.91; $0.72-1.15, p=0.43$ ), or any composite outcome examined were found, but the authors acknowledge methodologic differences between their report and the prior NRN study that might affect direct comparisons. Still, prolonged early antibiotic exposure increased the risk of fungal sepsis (aOR 4.95; 1.93-12.69, $p<0.001$ ) among infants not treated with $\mathrm{CMV}$, suggesting harm of prolonged early antibiotic treatment, particularly among the ELBWs who were less severely ill. Collectively, this study shows that we are making progress towards reducing prolonged early antibiotic exposure. It also confirms that many ELBWs are still exposed to prolonged early antibiotics, that this exposure is harmful, and the practice is highly variable between centers with similar patient acuity. What remains unclear is the incidence of true infection in this population that would substantiate prolonged antibiotics and why centers have such wide variability in practice.

Just because it's what's done, doesn't mean it's what's should be done.

-Cinderella

A major challenge for all physicians is to effectively reevaluate and refine one's knowledge throughout a career. Habits are hard to break. One habit of neonatal providers is the frequent use of empiric antimicrobials. For decades, medical students, residents, and fellows have been taught to treat any symptomatic baby, and 
especially a symptomatic preterm infant, with a course of antimicrobials. Many sound historical factors influenced teaching a high degree of suspicion for neonatal sepsis, and experienced providers would often describe antibiotic administration as a "better safe than sorry" philosophy. Over the past decade, great strides have been made towards reducing unnecessary antibiotic exposure in neonates, without increasing the incidence of missed infection, particularly for late-preterm and term infants ${ }^{4,5}$. Serial observation, rather than intervention, is an increasingly targeted approach even in the neonatal intensive care unit (NICU). Factors that are associated with low risk of infection in the extremely preterm population have been described ${ }^{6}$, yet the most preterm $(<29$ weeks) and the smallest (ELBW) infants still pose a significant daily clinical dilemma, generate high concern for infection among providers, and evoke habits that are hard to break.

Extremely preterm and ELBW infants exhibit significant physiologic immaturity and manifest respiratory distress that often requires invasive mechanical ventilation, as well as other non-specific signs that could also be the result of an infection. Discrimination of clinical signs that are the result of immaturity from those that are due to an infection is not always possible, and withholding effective empiric antimicrobials in the setting of infection would be irresponsible. Although the incidence of culture-proven early-onset bacteremia in this age group is the highest of all newborns, it occurs in only 3 of 100 infants born at $<29$ weeks $^{7}$. Decisions are made to treat or not treat based on perceived probability of the condition, the risk and effectiveness of the treatment, and the consequences of not acting. As a result, the overwhelming majority of these extremely preterm infants still receive early empiric antimicrobial treatment ${ }^{8}$. At present, the certainty that would allow providers to withhold initial empiric treatment for these infants is lacking. Diagnostic testing methods may eventually allow us to safely withhold the initiation of empiric antibiotics in this population ${ }^{9}$. Although efforts to establish a consensus definition of neonatal sepsis to align investigators and reduce variability are underway ${ }^{10}$, no consensus presently exists, which makes the criteria for this diagnosis, and its treatment decisions, highly variable and subjective.

A reduction of empiric antibiotic exposure beyond $48 \mathrm{~h}$ in 569 VLBW infants was feasible using serial C-reactive protein measures ${ }^{11}$. Opt-in approaches for continuation of antimicrobials beyond $48 \mathrm{~h}$ can be integrated into a NICU practice culture without the requirement for electronic health record automation. If a patient does not manifest evidence of non-specific inflammation on serial assessment as one would expect if infection were the cause of their clinical signs, especially without the identification of a pathogen, how can one reasonably justify prolonged antimicrobial treatment? Even the duration for culture-proven bacterial infections in this population, largely based on habits entrenched by our training and mentors, is not evidence based and is in desperate need of a precision medicine approach. As earlier work by Cordero and colleagues ${ }^{12}$, Cotten and colleagues ${ }^{1}$, and the present work by Greenberg and colleagues shows, broad exposure and wide variation in the incidence of prolonged early antibiotic exposure is unlikely to be the result of treating different patient populations, but rather a manifestation of local practice habits. These data show what is done, but it is not what should be done.

\section{ACKNOWLEDGEMENTS}

This work was supported by $\mathrm{NIH} / \mathrm{NICHD}$ (R01HD089939) and NIH/NIGMS (R01GM128452).

\section{ADDITIONAL INFORMATION}

Competing interests: The author declares no competing interests.

Publisher's note: Springer Nature remains neutral with regard to jurisdictional claims in published maps and institutional affiliations.

James L. Wynn ${ }^{1,2}$

${ }^{1}$ Department of Pediatrics, Division of Neonatology, University of Florida, 1600 SW Archer Road PO Box 100296, Gainesville, FL, USA and ${ }^{2}$ Department of Pathology, Immunology, and Laboratory Medicine, University of Florida, Gainesville, FL, USA Correspondence: James L. Wynn (james.wynn@peds.ufl.edu)

\section{REFERENCES}

1. Cotten, C. M. et al. Prolonged duration of initial empirical antibiotic treatment is associated with increased rates of necrotizing enterocolitis and death for extremely low birth weight infants. Pediatrics 123, 58-66 (2009).

2. Kuppala, V. S., Meinzen-Derr, J., Morrow, A. L. \& Schibler, K. R. Prolonged initial empirical antibiotic treatment is associated with adverse outcomes in premature infants. J. Pediatr. 159, 720-5 (2011).

3. Greenberg, R. G. et al. Prolonged duration of early antibiotic therapy in extremely premature infants. Pediatr Res. (2019). https://doi.org/10.1038/s41390-019-0300-4. [Epub ahead of print]

4. Kuzniewicz, M. W. et al. A quantitative, risk-based approach to the management of neonatal early-onset sepsis. JAMA Pediatr. 171, 365-71 (2017).

5. Benitz, W. E., Wynn, J. L. \& Polin, R. A. Reappraisal of guidelines for management of neonates with suspected early-onset sepsis. J. Pediatr. 166, 1070-4 (2015).

6. Puopolo, K. M. et al. Identification of extremely premature infants at low risk for early-onset sepsis. Pediatrics 140, pii: e20170925 (2017). https://doi.org/10.1542/ peds.2017-0925.

7. Stoll, B. J., Hansen, N. I. \& Bell, E. F. et al. Neonatal outcomes of extremely preterm infants from the NICHD Neonatal Research Network. Pediatrics 126, 443-56 (2010).

8. Flannery, D. D. et al. Temporal trends and center variation in early antibiotic use among premature infants. JAMA Netw. Open 1, e180164 (2018).

9. Sweeney, T. E. et al. Validation of the sepsis metascore for diagnosis of neonatal sepsis. J. Pediatr. Infect. Dis. Soc. 7, 129-35 (2018).

10. Wynn, J. L. \& Polin, R. A. Progress in the management of neonatal sepsis: the importance of a consensus definition. Pediatr. Res. 83, 13-15 (2018).

11. Coggins, S. A. et al. Use of a computerized C-reactive protein (CRP) based sepsis evaluation in very low birth weight (VLBW) infants: a five-year experience. PLoS ONE 8, e78602 (2013).

12. Cordero, L. \& Ayers, L. W. Duration of empiric antibiotics for suspected early-onset sepsis in extremely low birth weight infants. Infect. Control Hosp. Epidemiol. 24, 662-6 (2003). 